\title{
CORONAL AND HELIOSPHERIC OBSERVATIONS OF SOLAR PARTICLE EVENTS
}

\author{
MONIQUE PICK \\ Observatoire de Paris, DASOP, URA CNRS 1756, Meudon, 92195
}

September 23, 1996

\begin{abstract}
Observations of accelerated particle beams are used to probe the coronal and interplanetary magnetic field structures over large distances from the Sun on the order of a few $\mathrm{AU}$ and for various heliolatitudes. It is shown that the propagation of low energy particles is very much controled by discrete interplanetary magnetic field structures. These discrete magnetic structures are sometimes embedded within interplanetary solar wind plasma disturbances, commonly called CMEs. The connection between the corona and the interplanetary medium is discussed. These observations lead to new insights on the origin of accelerated particles detected in association with CMEs.
\end{abstract}

\section{Introduction}

It is well established that accelerated electron beams can be used to probe the coronal and interplanetary magnetic field structures over large distances from the Sun on the order of a few AU (see for example Anderson et al., 1992). These electron beams are detected either indirectly by the radio emission they produce along their trajectory path or by in-situ space probe measurements. In the present paper, we present observations of solar flare particle events. These observations show that:(a) The propagation of low energy particles is very much controled by discrete interplanetary magnetic field (IMF)/solar wind structures which can persist to large distances, at least several AU. Inside these structures, which are connected to specific locations in the corona, solar particles can propagate with little scattering. (b) Electron beams can be occasionally mirrored in magnetic field enhancements and remain inside the same propagating channel which carries electrons moving toward the Sun, as well as away from the Sun. (c) These discrete magnetic structures are sometimes embedded within interplanetary solar wind plasma disturbances (commonly called CMEs, see for the exact terminology Schwenn in this issue). Most of these observations were already published as individual cases in the literature. Here, we attempt to provide an overall view, on the large scale topology of the interplanetary magnetic field, in particular in the cases of interplanetary CMEs and we discuss the connection between the corona and the interplanetary medium. The interplanetary measurements reported here were made with the various experiments aboard the Ulysses probe (Balogh et al., 1992, Bame et al., 1992, Lanzerotti et al., 1992, Simpson et al., 1992, Stone et al., 1992); in particular, the low-energy 
particle data were obtained with the HI-SCALE instrument (Lanzerotti et al., 1992).

\section{Impulsive solar electron events and particle propagation channels}

Figure (1) exhibits a typical impulsive solar flare electron event that was channeled over an heliocentric distance of 4.3 AU (Buttighoffer et al., 1995). The propagation channel is identified in the solar wind and the magnetic field observations reported in Figure (1) (see thick grey bars). The pitch angle distribution measurements show that this event was nearly scatter free until about September 26, 23:00 UT. This is precisely the time at which Ulysses left the propagation channel. This impulsive electron event was associated with a solar type III burst at 06:04 UT on September 26 subsequently detected in the interplanetary medium by the URAP instrument aboard Ulysses. Langmuir waves inside the propagation channel are shown in Figure 1. The data analysis of this event reveals the existence of a magnetic structure which is anchored in the corona near the solar active region responsible for the electron acceleration and which maintains its identity to at least 4.3 AU. This structure of small dimension (about 10 millions of kilometers at $4 \mathrm{AU}$, i.e. $6000 \mathrm{~km}$ on the solar surface) is favorable to the free streaming of energetic electrons. Inside this structure the magnetic field is very quiet and one-dimensional with the minimum variance direction aligned with the mean magnetic field direction. Several other similar events were observed and all of them point out the existence of discrete and magnetically quiet structures with a coronal origin. They are nevertheless identified for different heliospheric conditions within the plasma physical environment. The next example (Anderson et al., 1995) is the solar impulsive electron event observed on February 22, 1991 and reported in Figure 2. The lower part of Figure 2 shows that the temporal evolution of the electron flux away from the sun is very much different compared to the evolution of the electrons toward the sun. The upper part of Figure 2 reproduces the Pitch Angle Distribution (PAD) obtained for two electron energy ranges at several times early in the event and shows that the electrons are seen highly collimated (the whole sky has been divided in 12 sectors, for more details, see Lanzerotti et al,1992). It is also seen that after 05:15 UT a return current is detected then vanishes and reappears at 05:47 UT. The PAD exhibits a loss cone indicated by dashed lines. Hence, this event provides evidence that fast electrons moving away from the sun are mirrored in magnetic field enhancements and return to their original observation point. Figure 3 gives a schematic representation of the geometry of the region crossed by Ulysses. From temporal and geometrical considerations, the distance between the mirror and the probe 

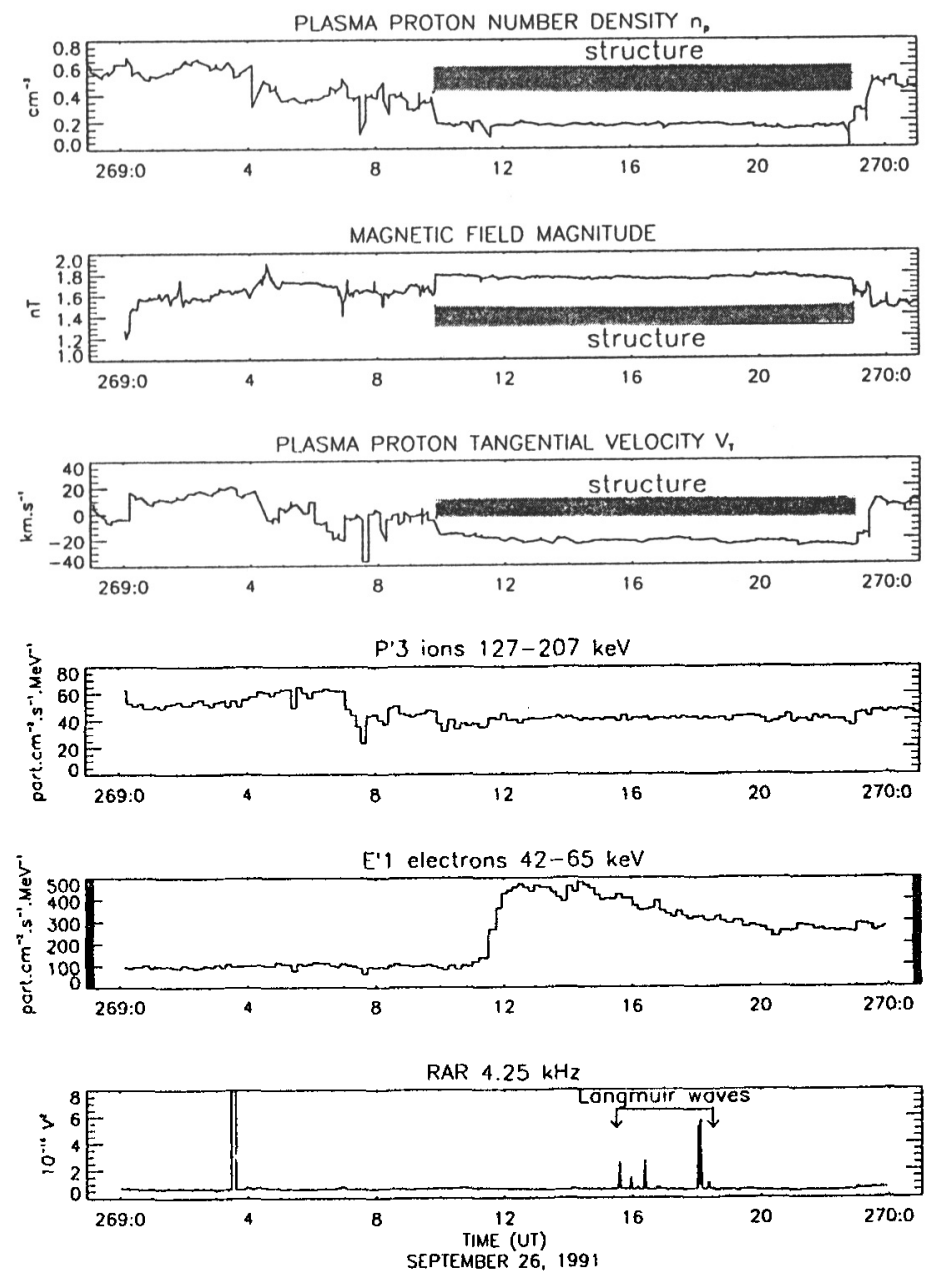

Fig. 1. September 26, 1991. Ulysses observations. From top to bottom: plasma density, resolution $30 \mathrm{~min}$; magnetic field magnitude, resolution $12 \mathrm{~s}$; plasma tangential velocity, resolution $30 \mathrm{~min}$; ion fluxes for $127-207 \mathrm{keV}$ energy range, resolution $15 \mathrm{~min}$, measured by one detector; electron fluxes for $42-65 \mathrm{keV}$ energy range, resolution $15 \mathrm{~min}$, measured by one detector; radio noise level at $4.25 \mathrm{kHz}$, resolution $128 \mathrm{~s}$. structure and Langmuir waves signatures are respectively indicated by thick grey bars and arrows.(From Buttighoffer et al., 1995.).

can be estimated, as well as the strength of the magnetic field at the mirror point. Furthermore, for this event, it was possible to identify the mirror which corresponded to the enhanced magnetic fields behind the shock which was associated with a co-rotating interaction region, some $0.7 \mathrm{AU}$ further distant from Ulysses along the interplanetary magnetic field. 


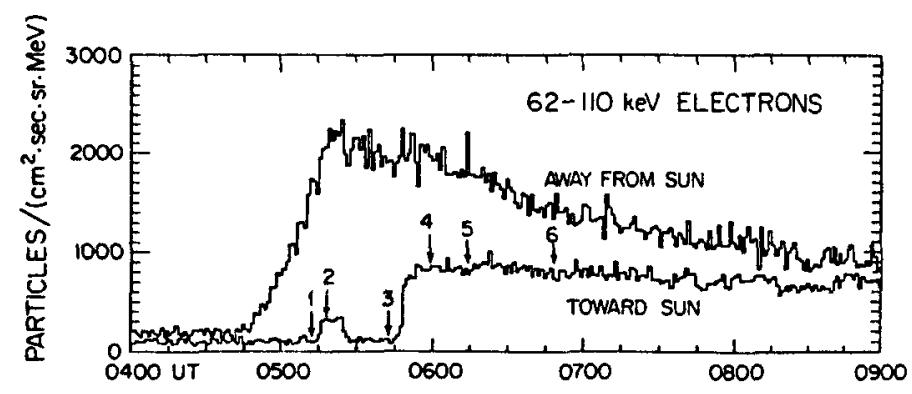

Fig. 2. Solar electron event on February 22, 1991 observed with Ulysses. Upper part: pitch angle distributions early in the solar flare electron event; counts are accumulated over $3 \mathrm{~min}$ for each distribution; evidence for a loss cone is seen in several examples; the critical angle for the loss cone is close to 37 . Lower part: temporal evolution of electron flux toward and away from the Sun for $62-110 \mathrm{keV}$ energy range.(From Anderson et al., 1995).

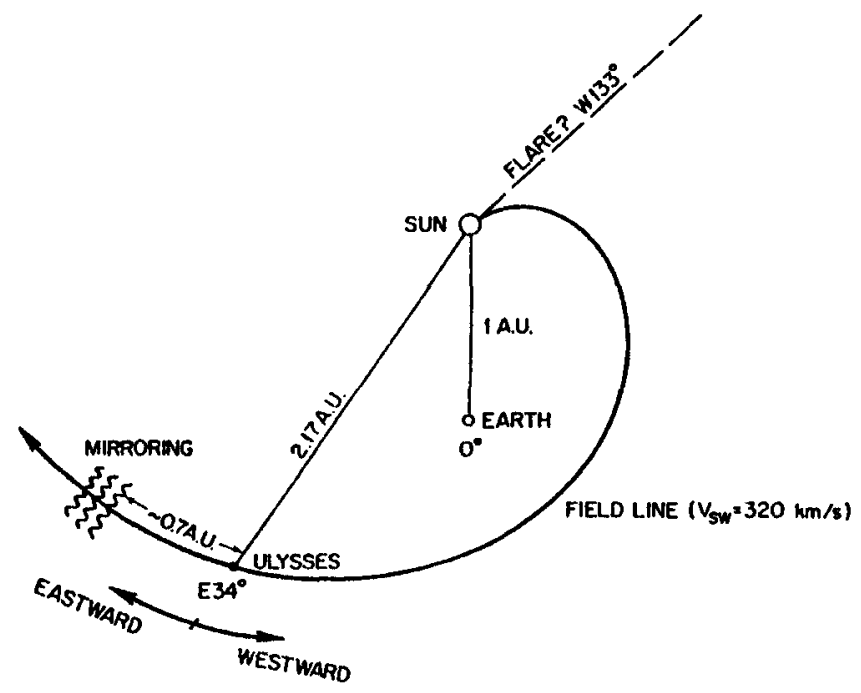

22 FEBRUARY 1991

Fig. 3. Schematic representation of the geometry of the region crossed by Ulysses at the time of the solar electron event on February 22, 1991.(From Anderson et al., 1995).

\section{Solar wind distrurbances (CMEs) and coronal particle propagation channels}

Particle propagation channels have been detected inside or at the boundary of solar wind disturbances (CMEs) (Armstrong et al., 1994; Pick et al., 1995) and in association with nearly scatter-free particle events. Hence, these observations imply important conclusions not only on the topological 


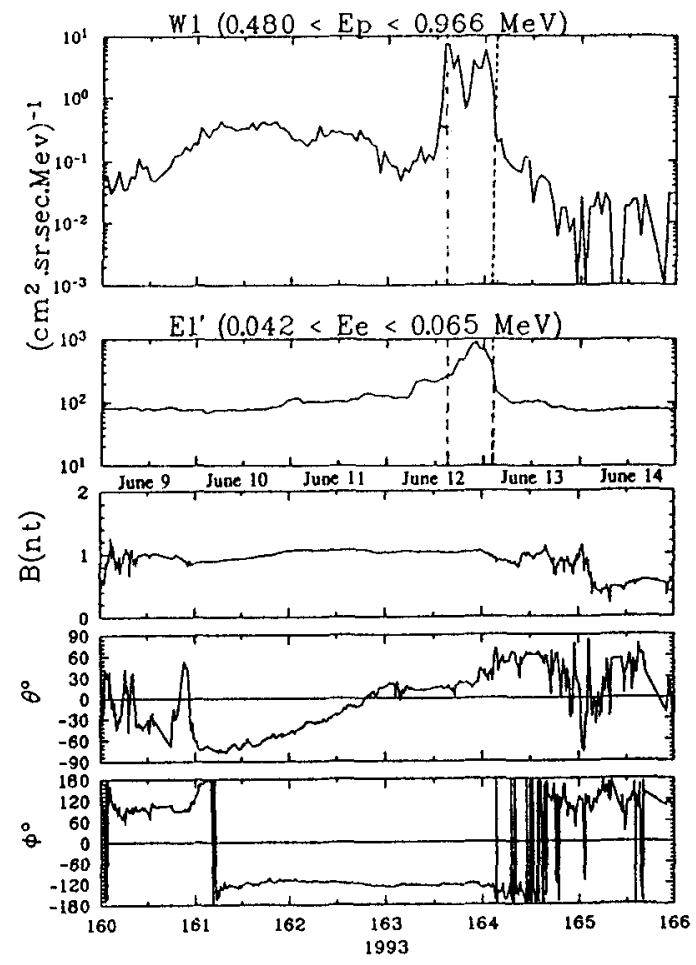

Fig. 4. June 9 (day 160) through June 14 (day 165), 1993 observed with the Ulysses instruments. From upper to lower pannel: spin and hourly-averaged fluxes of 0.480 to $0.966 \mathrm{MeV}$ protons, 0.042 to $0.065 \mathrm{MeV}$ electrons, IMF magnitude, latitude and azimuth angle (angle $=0$ points toward the Sun). (From Armstrong et al., 1994).

structure of CMEs in defining the connection of the corona to the interplanetary medium, but also on the origin and transport of fast particles detected inside CME. One of these events reported in Figure 4, occurred during the passage of a CME over the Ulysses spacecraft June 9-13, 1993 located at helioradius 4.6 AU and heliolatitude 32 south. On June 12 , the data show IMF-aligned streaming of $60 \mathrm{keV}$ to $2 \mathrm{MeV}$ ions directed away from the Sun .This ion beam has an energy spectrum peaked at $270 \mathrm{keV}$ and joint soft $\mathrm{X}$-ray, radio and particles data analysis demonstrated its coronal origin as illustrated in Figure 5. This figure shows the presence in the south hemisphere of one active region which during its passage across the solar disk was associated with solar radio noise storms indicating the presence of non thermal electrons. This active region was magnetically connected to Ulysses at the end of June 7 for a solar wind speed of about $700 \mathrm{~km} / \mathrm{s}$. On the same day, an important hard X-ray and radio event occurred in this region which presented all the required characteristics to produce the beam observed in the interplanetary medium. The absence of returning electron or ion current 


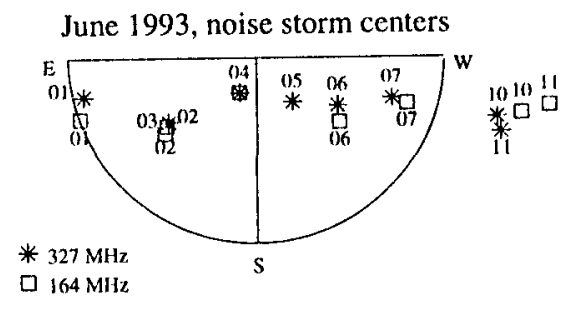

Fig. 5. Noise storms radio centers associated with AR 7518 during its passage on solar disk. These observations were obtained with the Nancay Radioheliograph. (From Pick et al., 1994).

suggests that this propagation channel from coronal origin detected inside the CME was open. Until recently, these CME were classically considered as connected to the Sun on both ends. This result is in agreement with Gosling et al. [1995] who have recently explored the magnetic topology of CMEs resulting from three-dimensional reconnection and found that open field lines embedded within some CMEs can be occasionally present. Another evidence of $\mathrm{CME}$ providing a conduit for energetic particles from coronal origin is illustrated in Figure 6 which shows the time evolution of a particle event observed on February 27, 1994 (Pick et al., 1995). This event occurred during the decreasing development of a large proton event. Shown in the lower panels of figure 6 are the spin average flux of protons and electrons in different energy ranges. The upper panel shows the IMF which became noticeably quiet on February 27 and 28, signalling the arrival at Ulysses of this CME.

\section{Conclusion}

All the events reported in the previous sections illustrate the value of using fast particles moving along the field lines to infer the large scale topology of interplanetary field lines and their connection with the corona. Moreover, these observations lead to new insights on the origin of accelerated particles detected in association with CMEs. They show that inside CMEs, particle injection from coronal origin can fill up a propagation channel and be effectively channeled, far from the accelerating site, over large heliocentric distances, without invoking any coronal diffusion process. For the June 1993 event, on one side, the radio observations provide evidence that non-thermal electrons are continuously accelerated in the corona during several days and that the region responsible for the noise storms is magnetically connected with the CME. On the other side, fast interplanetary electrons are also 


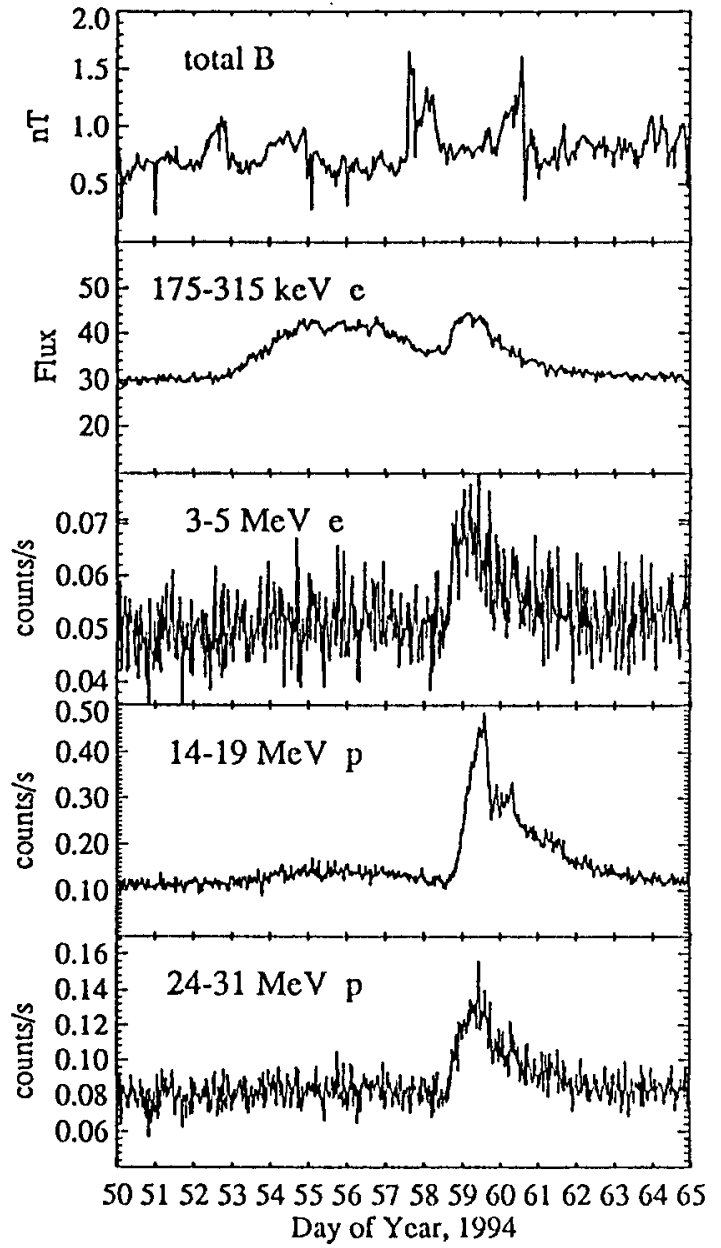

Fig. 6. Total magnitic field magnitude (Gosling et al., 1974), electron, and proton intensities versus time for the interval 19 February -5 March, 1994. (From Pick et al., 1995).

continuously detected inside the CME. These interplanetary electrons may either have been released from the corona or have been accelerated by the extended bow shock of a CME as proposed by Reames (1994) for the Solar Energetic Proton (SEP) events. Further studies are necessary to evaluate the respective importance of these two processes. We emphasize that for both interpretations long term storage is not necessary. 


\section{References}

Anderson, K.A., P.Chaizy, R.P. Lin and J.Sommers: 1992, Non relativistic solar electron events during December 1990: Results from Ulysses, Geophys. Res. lett., 19, 1283

Anderson, K.A., J.Sommers, R.P.Lin, M.Pick, P.Chaizy, N.Murphy, E.J.Smith, J.L.Phillips: 1995, Mirroring of fast solar flare electrons on a downstream co-rotating interaction region, J.Geophys.Res, 100, 3

Armstrong T.P., D. Haggerty, L. J. Lanzerotti, C. G. Maclennan, E. C. Roelof, M. Pick, G. M. Simnett, R. E. Gold, S. M. Krimigis, K. A. Anderson, R. P. Lin, E. T. Sarris, R. Forsyth, and A. Balogh: 1994, Observation by Ulysses of hot ( $270 \mathrm{keV}$ ) coronal particles at 32 degrees south heliolatitude and 4.6 AU, Geophys. Res. Lett., 21, 1747

Balogh A. and 7 coauthors: 1992, Astronomy and Astrophysics Suppl., 92, 221

Bame J. and 7 coauthors: 1992, Astronomy and Astrophysics Suppl., 92, 237

Buttighoffer A., M. Pick, E. C. Roelof, S. Hoang, A. Mangeney, L. J.Lanzerotti, R. J. Forsyth, and J. L. Phillips : 1994, Coronal electron stream and Langmuir wave detection inside a propagation channel at $4.3 \mathrm{AU}, J$. Geophys. Res, 100, A3, 3369

Gosling J.T. and 6 coauthors, A new class of forward-reverse shock pairs in the solar wind, Geophys. res. Letters, 21, 2271

Gosling J.T., J. Birn, M. Hesse: 1995, Three-dimensional magnetic reconnection and the magnetic topology of coronal mass ejection events, Geophysical Rsearch Letters, 22, 869

Lanzerotti L.J. and 10 coauthors: 1992, Heliosphere Instrument for spectra, composition and anisotropy at low energies, Astronomy and Astrophysics, Suppl., 92, 349

Pick M., A. Buttighoffer, A. Kerdraon, T.P. Armstrong, E.C. Roelof, S. Hoang, L.J. Lanzerotti, G.M. Simnett, J. Lemen : 1995, Ulysses observations of a coronal origin particle event at 32 south heliographic latitude, 1994, 28th ESLAB symposium, Space Science Reviews, 72, 1-2, 315

Pick M., L.J. Lanzerotti, A. Buttighoffer, E.T. Sarris, T.P. Armstrong, G.M. Simnett., E.C. Roelof , A. Kerdraon: 1995, The propagation of sub MeV solar particles to heliolatitudes above $50 \mathrm{~S}$, Geophys. Res. Lett., submitted

Reames D. V.: 1994, Solar energetic particles: a paradigm shift, Quadrennial U.S. national Report to the IUGG

Simpson J. A. and 30 coauthors: 1992, the Ulysses cosmic ray and solar particle investigation, Astronomy and Astrophysics Suppl, 92, 365

Stone R.G. and 31 coauthors: 1992, The unified radio and plasma wave investigation, Astronomy and Astrophysics, 92, 291 